\title{
Stabilization of the linear Kawahara equation
}

\author{
Carlos Frederico Vasconcellos, $\quad$ Patrícia N. da Silva \\ Depto de Análise Matemática, IME, UERJ, \\ 20550-013, Rio de Janeiro, RJ \\ E-mail: cfredvasc@ime.uerj.br, nunes@ime.uerj.br
}

Resumo: We study the stabilization of global solutions of the linear Kawahara equation (K) with periodic boundary conditions over the interval $(0,2 \pi)$ under the effect of a localized damping mechanism. The Kawahara equation is a model for small amplitude long waves. Using separation of variables, the Ingham inequality, multiplier techniques and compactness arguments we prove the exponential decay of the solutions of the $(K)$ model.

Palavras-chave: Kawahara equation, periodic boundary conditions, stabilization

In the Kawahara equation

$$
u_{t}+u_{x}+\kappa u_{x x x}+\eta u_{x x x x x}+u u_{x}=0,
$$

the conservative dispersive effect is represented by the term $\left(\kappa u_{x x x}+\eta u_{x x x x x}\right)$. This equation is a model for plasma wave, capilarity-gravity water waves and other dispersive phenomena when the cubic KdV-type equation is weak. Kawahara [8] pointed out that it happens when the coefficient of the third order derivative in the KdV equation becomes very small or even zero. It is then necessary to take into account the higher order effect of dispersion in order to balance the nonlinear effect. Kakutani and Ono [7] showed that for a critical value of angle between the magneto-acoustic wave in a cold collision-free plasma and the external magnetic field, the third order derivative term in the KdV equation vanishes and may be replaced by the fifth order derivative term. Following this idea, Kawahara [8] studied a generalized nonlinear dispersive equation which has a form of the KdV equation with an additional fifth order derivative term. This equation has also been obtainded by Hasimoto [6] for the shallow wave near critical values of surface tension. More precisely, in this work Hasimoto found these critical values when the Bond number is near to one third.

While analyzing the evolution of solutions of the water wave-problem, Schneider and Wayne [16] also showed that the coefficient of the third order dispersive term in nondimensionalized statements of the $\mathrm{KdV}$ equation vanishes when the Bond number is equal to one third. The Bond number is proportional to the strength of the surface tension and in the KdV equation it is related to the leading order dispersive effects in the water-waves problem. With its disappearance, the resulting equation is just Burger's equation whose solutions typically form shocks in finite time. Thus, if we wish to model interesting behavior in the water-wave problem it is necessary to include higher order terms. That is, it is necessary to consider the Kawahara equation. In any case, the inclusion of the fifth order derivative term takes into account the comparative magnitude of the coefficients of the third and fifth power terms in the linearized dispersion relation.

Berloff and Howard [2] presented the Kawahara equation as the purely dispersive form of the following nonlinear partial differential equation

$$
u_{t}+u^{r} u_{x}+a u_{x x}+b u_{x x x}+c u_{x x x x}+d u_{x x x x x}=0 .
$$

The above equation describes the evolution of long waves in various problems in fluid dynamics. The Kawahara equation corresponds to the choice $a=c=0$ and $r=1$ and describes water waves 
with surface tension. Bridges and Derks [5] presented the Kawahara equation - or fifth-order $\mathrm{KdV}$-type equation - as a particular case of the general form

$$
u_{t}+\kappa u_{x x x}+\eta u_{x x x x x}=\frac{\partial}{\partial x} f\left(u, u_{x}, u_{x x}\right)
$$

where $u(x, t)$ is a scalar real valued function, $\kappa$ and $\eta \neq 0$ are real parameters and $f\left(u, u_{x}, u_{x x}\right)$ is some smooth function. The form (1) occurs most often in applications and corresponds to the choice of $f$ in $(2)$ with the form $f\left(u, u_{x}, u_{x x}\right)=-\frac{u^{2}}{2}$.

As noted by Kawahara [8], we may assume without loss of generality that $\eta<0$ in (1). In fact, if we introduce the following simple transformations

$$
u \rightarrow-u, \quad x \rightarrow-x \text { and } t \rightarrow t
$$

we can obtain an equation of the form of equation (1) in which $\kappa$ and $\eta$ are replaced, respectively, by $-\kappa$ and $-\eta$.

Hagarus et al. pointed out that the Kawahara equation

$$
u_{t}=u_{x x x x x}-\varepsilon u_{x x x}+u u_{x}
$$

in which $\varepsilon$ is a real parameter models water waves in the long-wave regime for moderate values of surface tension, Weber numbers close to $1 / 3$; and that for such Weber numbers the usual description of long water waves via the Korteweg-de Vries (KdV) equation fails since the cubic term in the linear dispersion relation vanishes and fifth order dispersion becomes relevant at leading order, $\omega(k)=k^{5}+\varepsilon k^{3}$. Positive (resp. negative) values of the parameter $\varepsilon$ in (3) correspond to Weber numbers larger (resp. smaller) than 1/3. For further considerations see Topper-Kawahara [17].

Dispersive problems have been object of intensive research (see, for instance, the classical paper of Benjamin-Bona-Mahoni [1], Biagioni-Linares [3], Bona-Chen [4], Menzala et al. [12], Rosier [13], and references therein). Recently global stabilization of the generalized KdV system have been obtained by Rosier-Zhang [14] and Linares-Pazoto [10] studied the stabilization of the generalized KdV system with critical exponents. For the stabilization of global solutions of the Kawahara under the effect of a localized damping mechanism, see Vasconcellos and Silva [18, 19, 20].

For controllability problems involving dispersive systems, we can consider the work of RusselZhang [15] about the KdV system; the paper by Linares-Ortega [11], where the Benjamin-Ono equation has been analyzed and the paper of Zhang and Zhao [21] for the Kawahara equation.

This paper is devoted to study the stabilization of global solutions of the linear Kawahara equation $(\mathrm{K})$ with periodic boundary conditions over the interval $(0,2 \pi)$ under the effect of a localized damping mechanism, that is, we consider the following problem:

$$
\begin{cases}u_{t}+\beta u_{x}+\kappa u_{x x x}+\eta u_{x x x x x}+a(x) u=0 & x \in(0,2 \pi), t>0 \\ u(0, t)=u(2 \pi, t), & t>0 \\ u_{x}(0, t)=u_{x}(2 \pi, t), & t>0 \\ u_{x x}(0, t)=u_{x x}(2 \pi, t), & t>0 \\ u_{x x x}(0, t)=u_{x x x}(2 \pi, t), & t>0 \\ u_{x x x x}(0, t)=u_{x x x x}(2 \pi, t), & t>0 \\ u(x, 0)=u_{0}(x), & x \in(0,2 \pi)\end{cases}
$$

The parameter $\eta$ is a negative real number, $\kappa \neq 0, \beta \in\{0,1\}$ and $a \in L^{\infty}(0,2 \pi), a \geq 0$ a.e. in $(0,2 \pi)$ and $\operatorname{supp} a \subset(\alpha, \beta) \subset(0,2 \pi)$.

The total energy associated with the (4) system is defined by

$$
E(t)=\frac{1}{2} \int_{0}^{2 \pi}|u(x, t)|^{2} d x=\frac{1}{2}\|u(t)\|^{2} .
$$


Using the above boundary conditions we prove that

$$
\frac{d E}{d t}=\frac{\eta}{2}\left|u_{x x}(0, t)\right|^{2}-\int_{0}^{2 \pi} a(x)|u(x, t)|^{2} d x \leq 0, \quad \forall t>0 .
$$

So, $E(t)$ is a nonincreasing function of time. This paper is devoted to analyze the following questions: Does the energy $E(t) \rightarrow 0$ as $t \rightarrow \infty$ ? Is it possible to find a rate of decay of the energy?

Our main result is presented in the following theorem.

Theorem 1. There exist $c>0$ and $w>0$ such that the energy $E(t)$ associated to the system (4) satisfies:

$$
E(t) \leq c e^{-w t}\left\|u_{0}\right\|_{L^{2}(0,2 \pi)}^{2}
$$

for all $u_{0} \in L^{2}(0,2 \pi)$

To prove Theorem 1, we consider some auxiliary results. First we consider the problem:

$$
\begin{cases}v_{t}+\beta v_{x}+\kappa v_{x x x}+\eta v_{x x x x x}=0 & x \in(0,2 \pi), t>0 \\ v(0, t)=v(2 \pi, t), & t>0 \\ v_{x}(0, t)=v_{x}(2 \pi, t), & t>0 \\ v_{x x}(0, t)=v_{x x}(2 \pi, t), & t>0 \\ v_{x x x}(0, t)=v_{x x x}(2 \pi, t), & t>0 \\ v_{x x x x}(0, t)=v_{x x x x}(2 \pi, t), & t>0 \\ v(x, 0)=u_{0}(x), & x \in(0,2 \pi)\end{cases}
$$

For $T>0$, we use separation of variables and a suitable inequality due to Ingham (in the version presented by Komornik [9]) to show there exists a constant $C_{1}=C_{1}(T)>0$ such that

$$
\left\|u_{0}\right\|_{L^{2}(0,2 \pi)}^{2} \leq C_{1} \int_{0}^{T} \int_{\alpha}^{\beta}|v(x, t)|^{2} d x d t, \quad \forall(\alpha, \beta) \subset(0,2 \pi)
$$

After that, we consider the following problem:

$$
\begin{cases}w_{t}+\beta w_{x}+\kappa w_{x x x}+\eta w_{x x x x x}=-a(x) u(x, t) & x \in(0,2 \pi), t>0 \\ w(0, t)=w(2 \pi, t), & t>0 \\ w_{x}(0, t)=w_{x}(2 \pi, t), & t>0 \\ w_{x x}(0, t)=w_{x x}(2 \pi, t), & t>0 \\ w_{x x x}(0, t)=w_{x x x}(2 \pi, t), & t>0 \\ w_{x x x x}(0, t)=w_{x x x x}(2 \pi, t), & t>0 \\ w(x, 0)=0, & x \in(0,2 \pi)\end{cases}
$$

where $a \in L^{\infty}(0,2 \pi), a \geq 0$ a.e. in $(0,2 \pi)$ and $\operatorname{supp} a \subset(\alpha, \beta) \subset(0,2 \pi)$ and $u$ is the solution of problem (4)

For $T>0$, we use multiplier techniques to show there exists a constant $C_{2}=C_{2}(T)>0$ such that

$$
\left\|u_{0}\right\|_{L^{2}(0,2 \pi)}^{2} \leq C_{2} \int_{0}^{T} \int_{\alpha}^{\beta}|u(x, t)|^{2} d x d t, \quad \forall(\alpha, \beta) \subset(0,2 \pi)
$$

Finally, we combine previous results to prove that for $T>0$, there exists a constant $C_{3}=$ $C_{3}(T)>0$ such that

$$
\left\|u_{0}\right\|_{L^{2}(0,2 \pi)}^{2} \leq C_{3} \int_{0}^{T} \int_{\alpha}^{\beta}|u(x, t)|^{2} d x d t, \quad \forall(\alpha, \beta) \subset(0,2 \pi)
$$

where $u$ is the solution of problem (4).

Theorem 1 follows from the auxiliary results and semigroup properties. 


\section{Referências}

[1] T.B.Benjamin, J.L.Bona And J.J.Mahony, Model equations for long waves in nonlinear dispersive systems, Philos. Trans. Roy. Soc. London, Ser. A 272, 1972, 47-78.

[2] N.G.Berloff, And L.N. Howard Solitary and Periodic Solutions for Nonlinear Nonintegrable Equations, Studies in Applied Mathematics, 99, 1997, 1-24.

[3] H.A.Biagioni and F.Linares, On the Benney-Lin and Kawahara Equations, J. Math. Anal. Appl., (211), 1997, 131-152

[4] J.L.Bona And H.Chen, Comparision of model equations for small-amplitude long waves, Nonlinear Anal. 38, 1999, 625-647.

[5] T.J.Bridges And G. Derks, Linear instability of solitary wave solutions of the Kawahara equation and its generalizations. SIAM J.Math. Anal. (33), 6, 2002, 1356-1378.

[6] H.Hasimoto, Water waves, Kagaku, 40, 1970, 401-408 [Japanese].

[7] T. Kakutani And H. Ono, Weak Non-Linear Hydromagnetic Waves in a Cold CollisionFree Plasma, J. Phys. Soc. Japan, 26, 1969, 1305-1318.

[8] T.Kawahara, Oscillatory solitary waves in dispersive media, Phys. Soc. Japan 33, 1972, 260-264

[9] V.Komornik And P.Loreti, Fourier Series in Control Theory, Springer-Verlag, New York, 2005.

[10] F.Linares And A.F.Pazoto, On the exponential decay of the critical generalized Korteweg-de Vries with localized damping, Proc. Amer. Math. Soc. 135, (5), 2007, 15151522 .

[11] F.Linares and J.H.Ortega, On the controllability and stabilization of the Benjamin-Ono equation, ESAIM Control Optim. Calc.Var. 11, (2), 2005, 204-218.

[12] G.P.Menzala, C.F.Vasconcellos and E.Zuazua, Stabilization of the Korteweg-de Vries equation with localized damping, Quarterly Applied Math., vol. LX, 1, 2002, 111-129.

[13] L.Rosien, Exact boundary controllability for the Korteweg-de Vries equation on a bounded domain, ESAIM, Control. Optimization and Calculus of Variations, vol.2, 1997, 33-55.

[14] L.Rosier And B.Y.Zhang, Global Stabilization of the Generalized Korteweg-De Vries Equation Posed on a Finite Domain, SIAM Journal on Control and Optimization (45), 3, 2006, 927-956.

[15] D.L.Russell and B.Y.Zhang, Exact controllability and stabilization of the Korteweg-de Vries equation, Trans.Amer.Math.Soc., 348, (9), 1996, 1515-1522.

[16] G.Schneider and C.E.WAyne, The rigorous approximation of long-wavelenght capillarygravity waves, Arch.Rational Mech. Anal. 162, 2002, 247-285.

[17] J.Topper And T.Kawahara, Approximate equations for long nonlinear waves on a viscous fluid, J. Phys. Soc. Japan, (44), 1978, 663-666

[18] C.F. Vasconcellos and P.N. Silva, Stabilization of the Linear Kawahara Equation with localized damping, Asymptotic Analysis 58, (4), 2008, 229-252.

[19] C.F. Vasconcellos And P.N. Da Silva, Stabilization of the linear Kawahara equation with localized damping 58(4) (2008), 229-252, Asymptotic Analysis, 66, 2010, 119-124. 
[20] C.F. Vasconcellos And P.N. DA Silva, Stabilization of the Kawahara equation with Localized Damping, ESAIM Control Optim. Calc. Var. 17, (2011), 102-116.

[21] Zhang, Bing-Yu; Zhao, Xiangqing Control and stabilization of the Kawahara equation on a periodic domain. (English) Commun. Inf. Syst. 12, No. 1, 77-95 (2012). 